\title{
Variação da acuidade visual em pacientes jovens com ectopia lentis submetidos à cirurgia
}

\author{
Variation of visual acuity in young patients with ectopia lentis submitted \\ tosurgery
}

\author{
Mauro Waiswol ${ }^{1}$ \\ Suel Abujamra ${ }^{2}$ \\ Ralph Cohen $^{3}$ \\ Geraldo Vicente de Almeida ${ }^{4}$
}

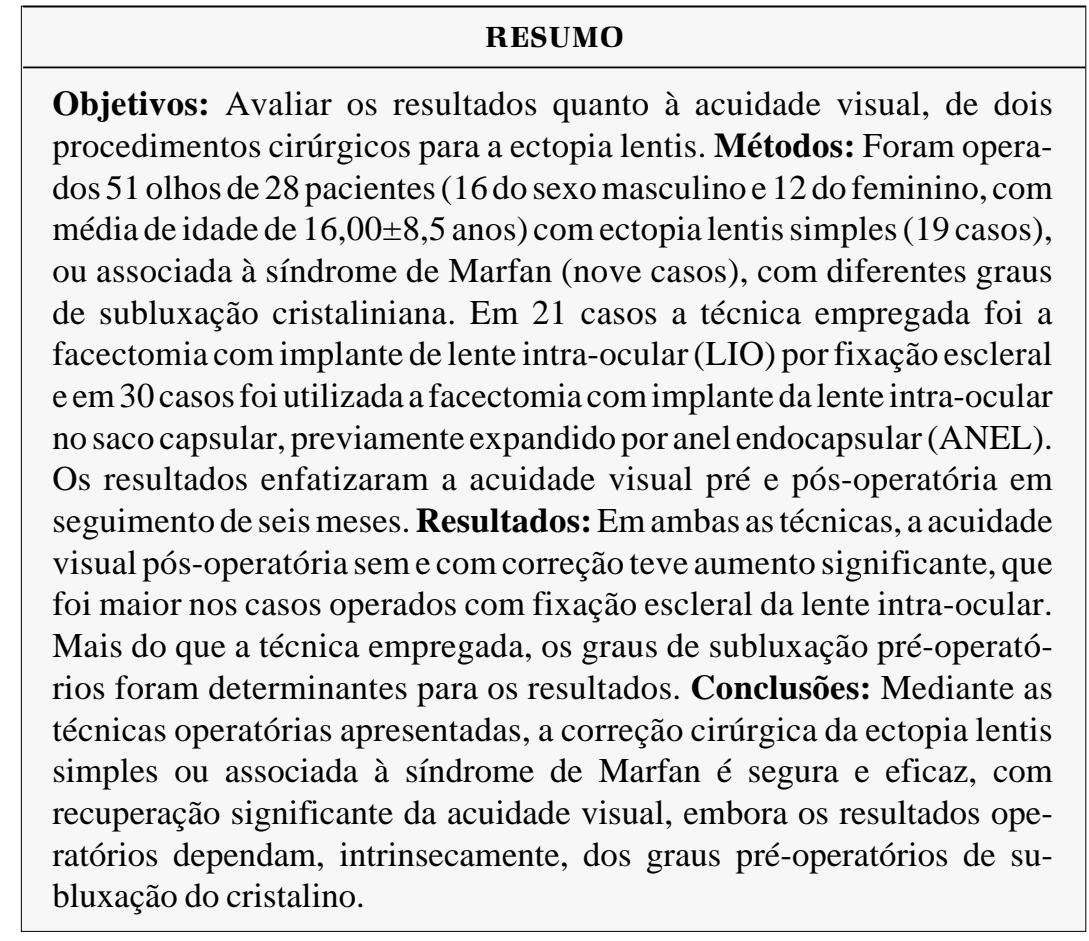

Descritores: Ectopia lentis/cirurgia; Síndrome de Marfan; Lentes intra-oculares; Esclera/ cirurgia; Cápsula do cristalino/cirurgia; Próteses e implantes; Acuidade visual; Adolescente

\section{INTRODUÇÃO}

Embora a ectopia lentis venha sendo tratada cirurgicamente há 140 anos, a conduta terapêutica a ser indicada, incluindo o momento ideal e a técnica operatória a ser empregada, é objeto de controvérsia, devido ao elevado número de complicações. Recentemente, com as modernas técnicas de microcirurgia e novos equipamentos ${ }^{(1-8)}$, a remoção do cristalino passou a ser mais claramente indicada para os casos cujo prejuízo visual não seja corrigível por refração, ou nos quais ocorrem complicações.

A correção óptica imediata e adequada dos erros refrativos nestes pacientes é essencial para a prevenção da ambliopia, durante o período de plasticidade do desenvolvimento visual. Nos casos em que o cristalino apresenta pequeno deslocamento, porém estável, o erro refrativo pode ser pequeno e facilmente corrigido com óculos ou lentes de contato. Quando não existem outras alterações, a maioria destes pacientes não deverá ser submetida à cirurgia ${ }^{(9-13)}$. 
Por outro lado, o deslocamento do cristalino pode produzir efeitos ópticos, cuja correção com óculos ou lentes de contato pode ser difícil, devido ao elevado grau de astigmatismo, bem como pela irregularidade da forma e rotação sobre seu eixo, em virtude da perda parcial das zônulas. Uma associação freqüente refere-se à miopia de grau elevado, que pode ser decorrente da ausência parcial das zônulas, o que resulta em miopia lenticular ${ }^{(14)}$.

O caso de ectopia lentis onde a indicação cirúrgica é mais comum é a bissecção da pupila pelo cristalino - equador do cristalino no eixo visual ${ }^{(11)}$. Nesta situação, mesmo que sejam possíveis a refração e a correção da visão para ambas as pupilas - a fácica e a afácica - a visão obtida ainda será problemática, em razão da função da interface entre as partes fácica e afácica na pupila, que reduz o contraste causando ofuscamento.

Algumas vezes, o cristalino é tão móvel, que as mudanças do erro refrativo se alteram continuamente, podendo evoluir para uma luxação completa ${ }^{(8)}$.

Assim, quando não é possível alcançar boa visão com correções refracionais cuidadosas e repetidas, quando o desempenho das atividades diárias é afetado, ou quando surgem sinais iminentes de complicações, a indicação cirúrgica deverá ser considerada ${ }^{(15-24)}$.

Didaticamente, as técnicas operatórias disponíveis hoje para a correção da ectopia lentis incluem: 1) a extração intracapsular; 2) a extração extracapsular; 3) a lensectomia com vitreófago; 4) a discissão-aspiração; e 5) a facoemulsificação.

O objetivo deste estudo foi comparar os resultados relativos à acuidade visual de dois procedimentos operatórios: a facectomia com implantação de lente intra-ocular (LIO) com fixação escleral e a implantação da LIO no saco capsular previamente expandido por anel endocapsular (ANEL).

\section{MÉTODOS}

Com a devida aprovação dos Comitês de Ética da Faculdade de Ciências Médicas da Santa Casa de São Paulo e da Faculdade de Medicina da Universidade de São Paulo, este estudo prospectivo foi realizado em 51 olhos de 28 pacientes ( 16 do sexo masculino e 12 do feminino, com idades entre $5,4 \mathrm{e}$ 37,6 anos e média de idade de $16,00 \pm 8,5$ anos, sem diferença significativa entre os sexos), todos com ectopia lentis simples (19 casos) ou associada com a síndrome de Marfan (9 casos), e tratados cirurgicamente pelo autor. Os pacientes foram selecionados do Ambulatório do Setor de Catarata do Departamento de Oftalmologia da Santa Casa de São Paulo e tinham indicação cirúrgica por visão suficientemente baixa para interferir na sua rotina diária (trabalho, estudo, lazer, afazeres domésticos etc.) e pelo interesse dos pacientes (ou dos pais dos pacientes) de submeter-se ao tratamento cirúrgico.

Para a inclusão dos pacientes neste estudo, considerou-se: 1) a presença de ectopia lentis em pelo menos um dos olhos; 2) acuidade visual, com a melhor correção, igual ou inferior a 0,4 ; 3) o consentimento dos pacientes (ou seus pais e/ou responsá- veis) após esclarecimento adequado sobre o estudo; e 4) a possibilidade de retorno dos pacientes para os exames de seguimento por pelo menos seis meses após a cirurgia.

Os critérios de exclusão para o estudo incluíram condições como: 1) luxação do cristalino para a câmara anterior ou posterior; 2) antecedentes de trauma ocular; 3) ceratopatias que impedissem o exame óptico adequado; 4) presença de glaucoma; 5) uveíte; 6) anormalidades retinianas que comprometessem a recuperação visual ou implicassem em risco aumentado de descolamento retiniano; 7) cirurgia oftalmológica prévia; 8) falta de condições clínicas gerais do paciente, conforme orientação do anestesista, caracterizadas por hipertensão arterial descompensada, cardiopatia grave ou outras doenças sistêmicas que implicassem em risco anestésico importante; e 9) falta de cooperação do paciente (ou seja, pacientes que não permitiram a realização adequada do exame; os que não poderiam retornar para seguimento, de acordo com o protocolo do estudo; e aqueles que provavelmente não fariam uso adequado da medicação prescrita).

Em 23 casos (82,1\%), a operação foi bilateral; em quatro casos $(14,3 \%)$ foi operado apenas o olho esquerdo; e em um caso $(3,6 \%)$ foi operado apenas o olho direito. Portanto, do total de 51 olhos operados, $27(52,9 \%)$ referiam-se ao lado esquerdo e $24(47,1 \%)$, ao lado direito.

Para determinar o grau de subluxação, elaboramos uma classificação quantitativa pessoal ${ }^{(25)}$ para mensuração objetiva do grau de deslocamento do cristalino em relação à área pupilar, sem uso de qualquer midriático. Para tanto, os pacientes foram examinados com lâmpada de fenda Haag Streit, inicialmente com pequeno aumento, iluminação difusa e fenda larga, de modo a se obter visão panorâmica. Para localização exata do cristalino, foi necessário recorrer à biomicroscopia com fenda bem estreita e com braço da lâmpada de fenda formando ângulo de $45^{\circ} \mathrm{com}$ o microscópio. A avaliação era completada com o campo vermelho. Os resultados dessa avaliação foram classificados em quatro graus de intensidade (Figura 1):

Grau 1: cristalino ocupando toda a área pupilar;

Grau 2: cristalino presente em dois terços da área pupilar;

Grau 3: cristalino presente em metade da área pupilar;

Grau 4: cristalino ausente da área pupilar.

Assim, quanto ao grau de subluxação do cristalino, 10 olhos (19,6\%) foram classificados em Grau 1, 26 olhos (51,0\%) em Grau 2 e 15 olhos $(29,4 \%)$ em Grau 3. Nenhum caso foi classificado com Grau 4 de subluxação.

As cirurgias foram realizadas no período de março de 2000 a junho de 2001, no Centro Cirúrgico Ambulatorial do Departamento de Oftalmologia da Santa Casa de Misericórdia de São Paulo, e o acompanhamento pós-operatório foi de seis meses, considerando-se seis avaliações realizadas no segundo e nono dias, primeiro, segundo, terceiro e sexto meses após a cirurgia.

A análise dos resultados enfatizou prioritariamente a acuidade visual sem (avaliada em todas as visitas pós-operatórias) e com (avaliada na última visita) correção, medida com o uso do "E" da tabela de Snellen a seis metros de distância e, não sendo possível a leitura, com aproximação progressiva do 


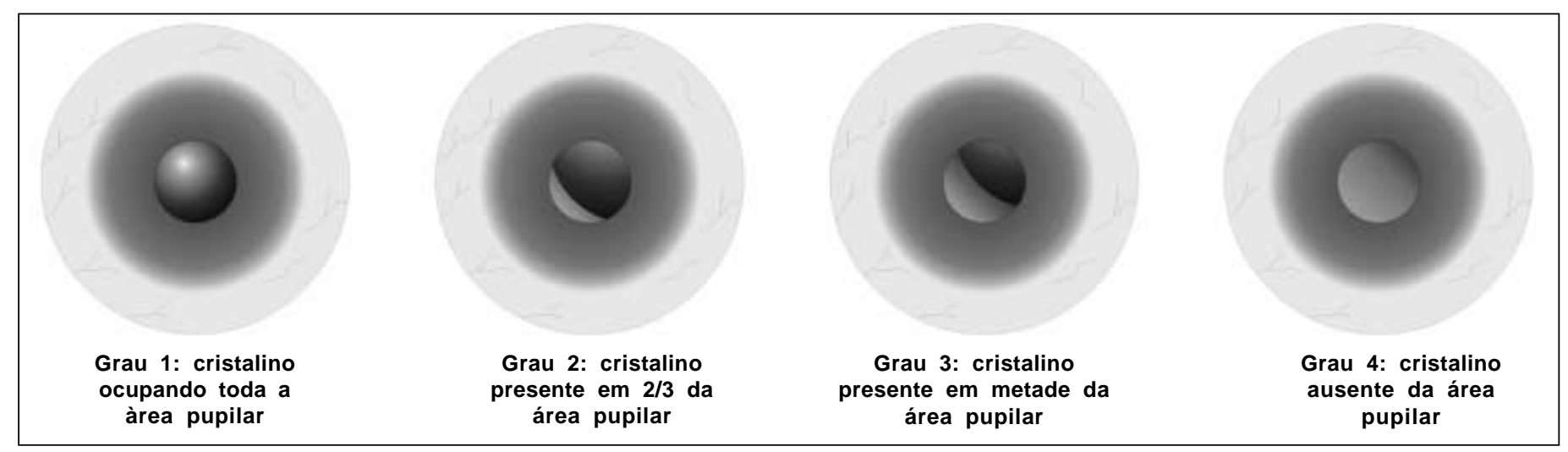

Figura 1 - Graus de subluxação

paciente à tabela, até obter-se a informação, convertendo-se, então, o valor encontrado de acordo com a distância necessária para leitura.

Os resultados foram analisados predominantemente por estatística paramétrica e, eventualmente, por estatística não paramétrica, cuja finalidade foi a de reforçar a significância dos dados encontrados.

Para todas as estatísticas utilizadas, selecionadas de acordo com a amostra analisada e respectivas variáveis, adotou-se nível de significância de $5 \%(\alpha=0,05)$.

\section{TÉCNICAS OPERATÓRIAS}

Após assepsia local com iodopovidine a $10 \%$ e colocação de campos cirúrgicos estéreis, realiza-se o posicionamento e a colocação de blefarostato. O músculo reto superior é fixado com pinça de reto superior e fio de seda 4-0, e realiza-se retalho conjuntival com base no fórnix de aproximadamente 90 graus superiormente. Procede-se à hemostasia dos vasos límbicos e perilímbicos e, então, à incisão em forma de "bocarra" (frown incision) de $5 \mathrm{~mm}$, com aproximadamente metade da espessura escleral, localizada a $2 \mathrm{~mm}$ do limbo cirúrgico. Para a incisão, utiliza-se lâmina de bisturi número 15 e inclinação de 90 graus em relação à esclera, procurando manter, quando possível, fidelidade ao eixo da zônula intacta (entre 3:00h e 9:00h).

É construído um túnel por delaminação escleral com bisturi descartável de lâmina crescente (modelo Crescent Knife, Satin Crescent, Angled, Bevel up, Alcon Surgical), que se estende do sulco escleral em direção à córnea, entrando $1,5 \mathrm{~mm}$ no estroma corneal, com metade da espessura corneal, obtendo-se incisão auto-selante. A incisão e entrada na câmara anterior são feitas com bisturi descartável de lâmina de 2,75 mm (modelo Slit Knife, Clear Cut, 2.75 mm Angled, Alcon Surgical), pela qual se introduz componente viscoelástico (Metilcelulose 2\%, Ophtalmos) até o aprofundamento da câmara anterior. Realizam-se paracenteses corneais a $1 \mathrm{~mm}$ do limbo, na posição correspondente às 9:00h e às 3:00h, com aproximadamente $1,5 \mathrm{~mm}$ de extensão, com bisturi descartável de lâmina de $1 \mathrm{~mm}$ (modelo I-Knife, Cutting Instrument $15^{\circ}$, Alcon Surgical).
Procede-se, então, à capsulotomia circular contínua de aproximadamente 4,0 $\mathrm{mm}$ de diâmetro (capsulorrexe), através da paracentese, com agulha $13,5 \times 4$, com a extremidade angulada, e complementando com pinça de Utrata através do túnel escleral. Nos casos em que é, de fato, possível obter a capsulotomia circular contínua, implanta-se anel endocapsular (ou anel expansor capsular) de polimetilmetacrilato de $12,5 \mathrm{~mm}$ (compressível até 10,0 mm, produzido pela Mediphacos) no saco capsular com o auxílio de injetor (produzido pela Morcher). Após a implantação do anel, realiza-se a hidrodissecção gentil com cânula (modelo K7-5054, Katena Eye Instruments), através da paracentese, seguida de aspiração "seca" do córtex cristaliniano, técnica que envolve a aspiração do material cristaliniano simultaneamente à introdução de metilcelulose $2 \%$ no saco capsular, e posterior aspiração, em sucessivas vezes, até a completa remoção do córtex cristaliniano com cânula (Anis) com orifício de 0,4 mm (Katena Eye Instrument). Introduz-se componente viscoelástico (Metilcelulose 2\%, Ophtalmos) na câmara anterior, e amplia-se a válvula corneal para a direita e para a esquerda, paralelamente ao limbo, até que atinja a mesma dimensão da incisão escleral ( $5 \mathrm{~mm}$ ). Implantase a LIO de câmara posterior modelo Slim (Mediphacos), biconvexa, de peça única de polimetilmetacrilato, com diâmetro de zona óptica de 5,0 mm-6,0 mm, A-constante de 118,3 e comprimento de alça a alça de $13,5 \mathrm{~mm}$. Procede-se ao centramento do saco capsular por meio da fixação escleral do ANEL e da LIO com fio de polipropileno 10-0 (prolene 10-0), monofilamento azul, com agulha reta de $16 \mathrm{~mm}$. A primeira agulha é dirigida através da incisão em direção ao quadrante com fraqueza zonular, pela abertura da capsulorrexe, passando por baixo do anel e do háptico da LIO, através do equador do saco capsular, até encontrar com a agulha $13 \times 4,5$ (Bene, Dicson), que atua como guia até a saída na esclera através do sulco ciliar a 1,5 mm do limbo. Previamente deve ser realizada peritomia conjuntival com extensão de aproximadamente $3 \mathrm{~mm}$, base no fórnix, retalho escleral triangular perilímbico. A outra extremidade do fio de prolene 10-0, também agulhado, é dirigida através da incisão e passa por fora do saco capsular, abaixo da íris, até atingir a agulha $13 \times 4,5$ (Bene, Dicson), que serve de 
guia até a saída na esclera, através do sulco ciliar a $1,5 \mathrm{~mm}$ do limbo cirúrgico. As agulhas são destacadas do fio, e as suturas são tracionadas, até que o saco capsular esteja centrado, quando é feito o anodamento seguido, por sua vez, da reposição e sutura do retalho escleral, com um ponto interrompido de náilon monofilamentado 10-0, sepultado no leito escleral e coberto com conjuntiva. A incisão escleral de $5 \mathrm{~mm}$ é suturada com dois pontos interrompidos de náilon monofilamentado 10-0, sepultados no leito escleral e cobertos com conjuntiva.

Nos casos em que a capsulotomia circular contínua não é obtida com o padrão técnico ideal, o ANEL não é implantado. Realiza-se, então, hidrodissecção gentil com cânula de hidrodissecção (modelo K7-5054, Katena Eye Instruments), através da paracentese, seguida de aspiração "seca" do córtex cristaliniano, ocasião em que se injeta metilcelulose $2 \%$ (Ophtalmos) no saco capsular e na câmara anterior, para o tamponamento do humor vítreo na área de fragilidade zonular, e se aspira até a completa remoção do córtex cristaliniano, em sucessivas vezes, com cânula de aspiração de córtex (Anis) com orifício de 0,4 mm (Katena Eye Instruments). Introduz-se componente viscoelástico (metilcelulose $2 \%$, Ophtalmos) na câmara anterior e, na seqüência, procede-se à ampliação da válvula corneal para a direita e para a esquerda, paralelamente ao limbo, até que atinja a mesma dimensão da incisão escleral de $7 \mathrm{~mm}$. A LIO modelo SF-70H (Mediphacos), biconvexa, de peça única de polimetilmetacrilato, com diâmetro de zona óptica de $7 \mathrm{~mm}$, A-constante de 118,3 e comprimento de alça a alça de $13,5 \mathrm{~mm}$, é suturada na esclera, usando-se fio de polipropileno 10-0 (prolene 10-0), monofilamento azul, com duas agulhas retas de $16 \mathrm{~mm}$. Os pontos de entrada são feitos a $1,5 \mathrm{~mm}$ posteriores ao limbo e a 1,0 $\mathrm{mm}$ acima ou abaixo do meridiano das 3 e 9 horas. A agulha reta passa através do leito de um retalho escleral, de forma triangular, até encontrar com a agulha $13 \times 4,5$ (Bene, Dicson), colocada através do leito do retalho escleral oposto, que atua como guia até a saída na esclera, através do sulco ciliar, a aproximadamente 1,5 $\mathrm{mm}$ do limbo. $\mathrm{O}$ mesmo procedimento é realizado no sentido oposto. $\mathrm{O}$ fio de sutura é exteriorizado pela incisão principal com o auxílio de um gancho de Lester, e é seccionado; suas extremidades são anodadas ao orifício contido no háptico da LIO. Introduz-se componente viscoelástico (metilcelulose $2 \%$, Ophtalmos) na câmara anterior, e amplia-se a válvula corneal para a direita e para a esquerda, paralelamente ao limbo, até que atinja a dimensão da incisão escleral de $7 \mathrm{~mm}$. A LIO é introduzida no olho, rodada na posição, e centrada através do ajuste da tensão da sutura. Uma vez que a LIO é adequadamente centrada, a sutura externa é anodada. Cada um dos retalhos esclerais é suturado com um ponto interrompido de náilon monofilamento 10-0, sepultado no leito escleral e coberto com conjuntiva. A incisão escleral de $7 \mathrm{~mm}$ é suturada com três pontos interrompidos de náilon monofilamento $10-0$, sepultados no leito escleral e cobertos com conjuntiva.

Realiza-se injeção subconjuntival de garamicina e dexametasona (1 ml) na região superior. Procede-se, por fim, à colocação de apósito ocular.
Na figura 2 encontram-se esquematizados os tempos operatórios, seguidos das respectivas ilustrações (Figuras 3 e 4).

Como tratamento pós-operatório, prescreveu-se o uso de colírio de tobramicina $3 \mathrm{mg}$ e dexametasona $1 \mathrm{mg}$ (Tobradex Alcon) seis vezes por dia, na primeira e segunda semanas; quatro vezes por dia, na terceira semana; três vezes por dia, na quarta semana, e redução gradual até a retirada. $\mathrm{O}$ uso de colírios midriáticos e antiinflamatórios sistêmicos foi reservado para os casos de reação inflamatória intra-ocular intensa.

\section{RESULTADOS}

Trinta olhos (58,8\%) foram operados com colocação de ANEL e LIO, e os $21(41,2 \%)$ restantes, com fixação escleral de LIO.

Quanto aos valores médios da acuidade visual sem correção dos 51 olhos estudados (Tabela 1), observa-se que, na avaliação pré-operatória, média e desvio padrão são semelhantes. Nas avaliações pós-operatórias, o desvio padrão decresce proporcionalmente para, no $180^{\circ}$ dia pós-operatório, atingir valor correspondente à aproximadamente metade do valor da média. O gráfico 1 representa a evolução da acuidade visual sem correção, no período pré-operatório e ao longo do pós-operatório, evidenciando elevação acentuada do valor médio da acuidade visual sem correção, até aproximadamente o $90^{\circ}$ dia pós-operatório, a partir de quando estes valores tendem à estabilização até o $180^{\circ}$ dia de pós-operatório, ainda apresentando, todavia, pequena ascensão.

A análise de variância dos valores médios de acuidade visual sem correção, desde o período pré-operatório até o $180^{\circ}$ dia de pós-operatório, mostrou variação conjunta significante ( $\mathrm{F}=90,19, \mathrm{p}<0,000001)$. A análise da variação das médias, par a par, em todas as 21 combinações possíveis, foi realizada com o emprego do teste post-hoc de comparação múltipla de Tukey, cujos resultados evidenciaram variação significante $(p<0,05)$ em 18 pares. Não foram significantes as variações destes

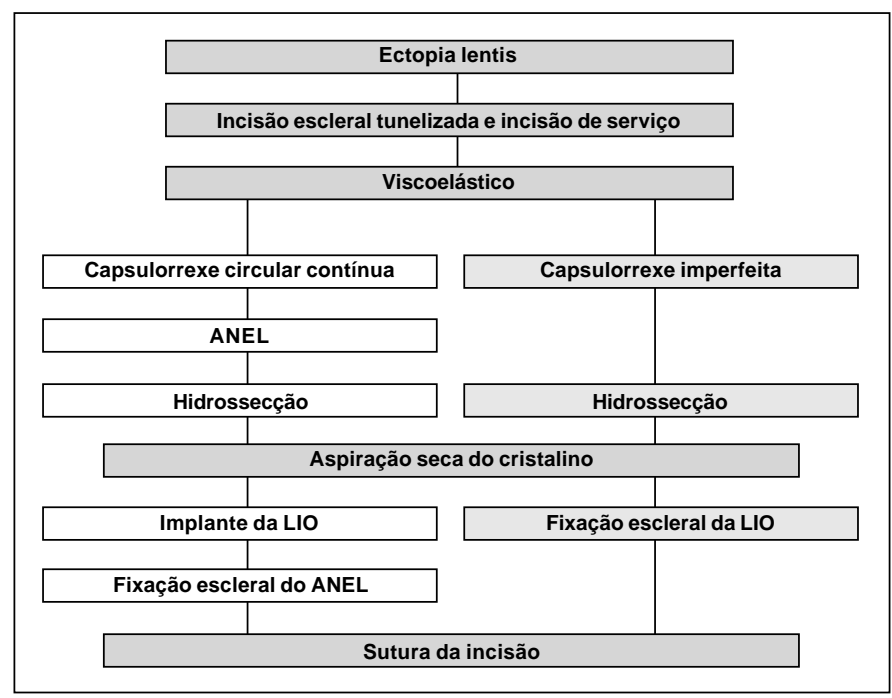

Figura 2 - Esquema dos tempos cirúrgicos 


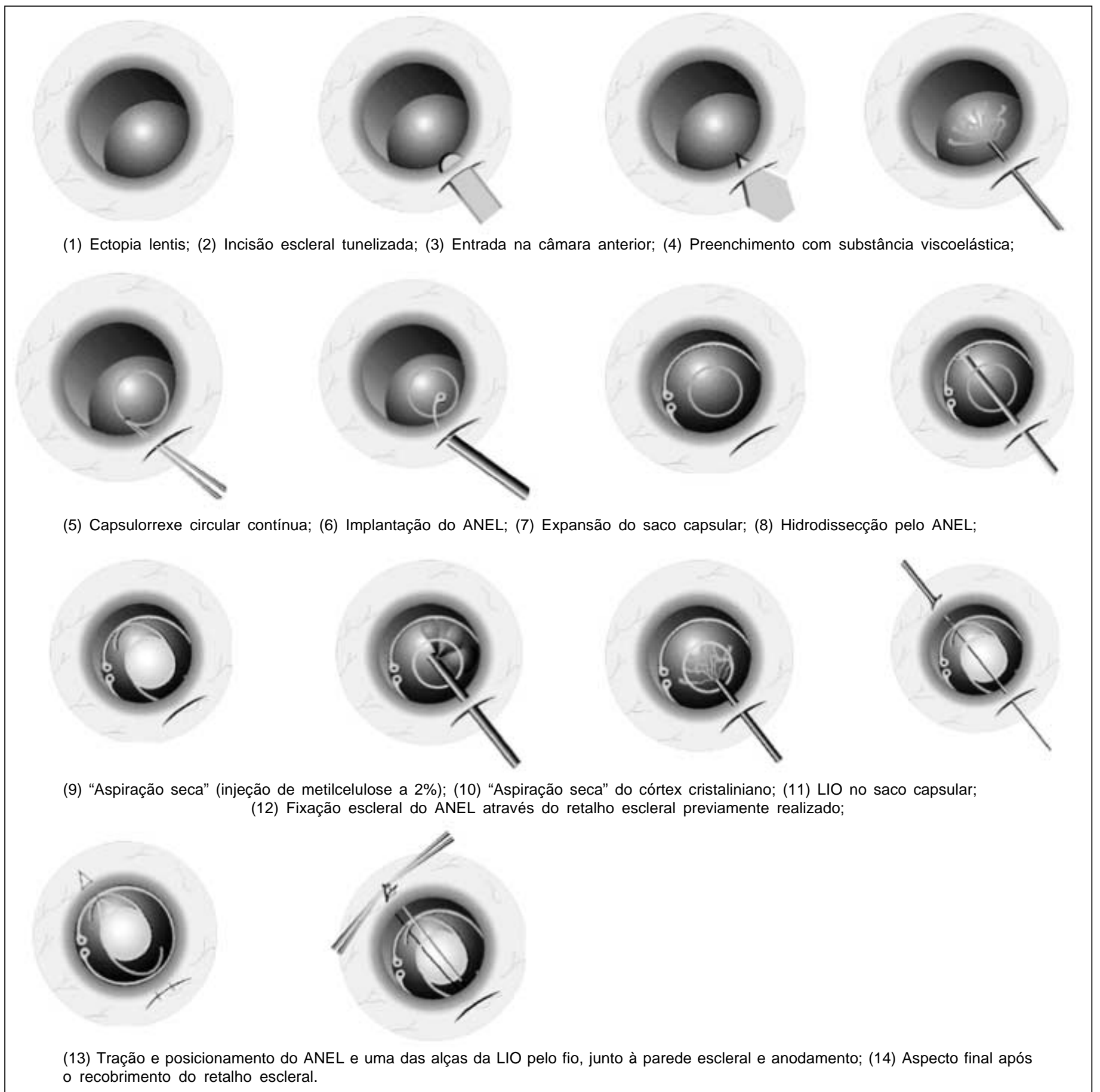

Figura 3 - Colocação do ANEL e da lente intra-ocular (LIO)

valores no pré-operatório versus o $2^{0}$ dia de pós-operatório $(\mathrm{p}=0,098307)$; no $60^{\circ}$ dia versus o $90^{\circ}$ dia pós-operatório $(\mathrm{p}=0,081903)$; nem no 90ํ dia versus o 180ํ dia pós-operatório $(\mathrm{p}=0,810978)$.

Houve variação significante com relação aos valores médios de acuidade visual com correção entre a avaliação préoperatória $(0,1795 \pm 0,1177)$ e a avaliação realizada no $180^{\circ}$ dia após a cirurgia $(0,6257 \pm 0,2662)$.
As maiores médias relativas à acuidade visual sem e com correção foram registradas para os olhos em que se utilizou a colocação de ANEL e LIO, em comparação com os olhos que tiveram a LIO fixada, tanto no pré-operatório quanto ao longo de todo o seguimento pós-operatório (Tabelas 2 e 3), e estas diferenças foram estatisticamente significantes (Gráfico 2).

Todavia, quando se verifica a variação entre os valores iniciais (pré-operatório) e os valores finais (180ํ dia pós-ope- 


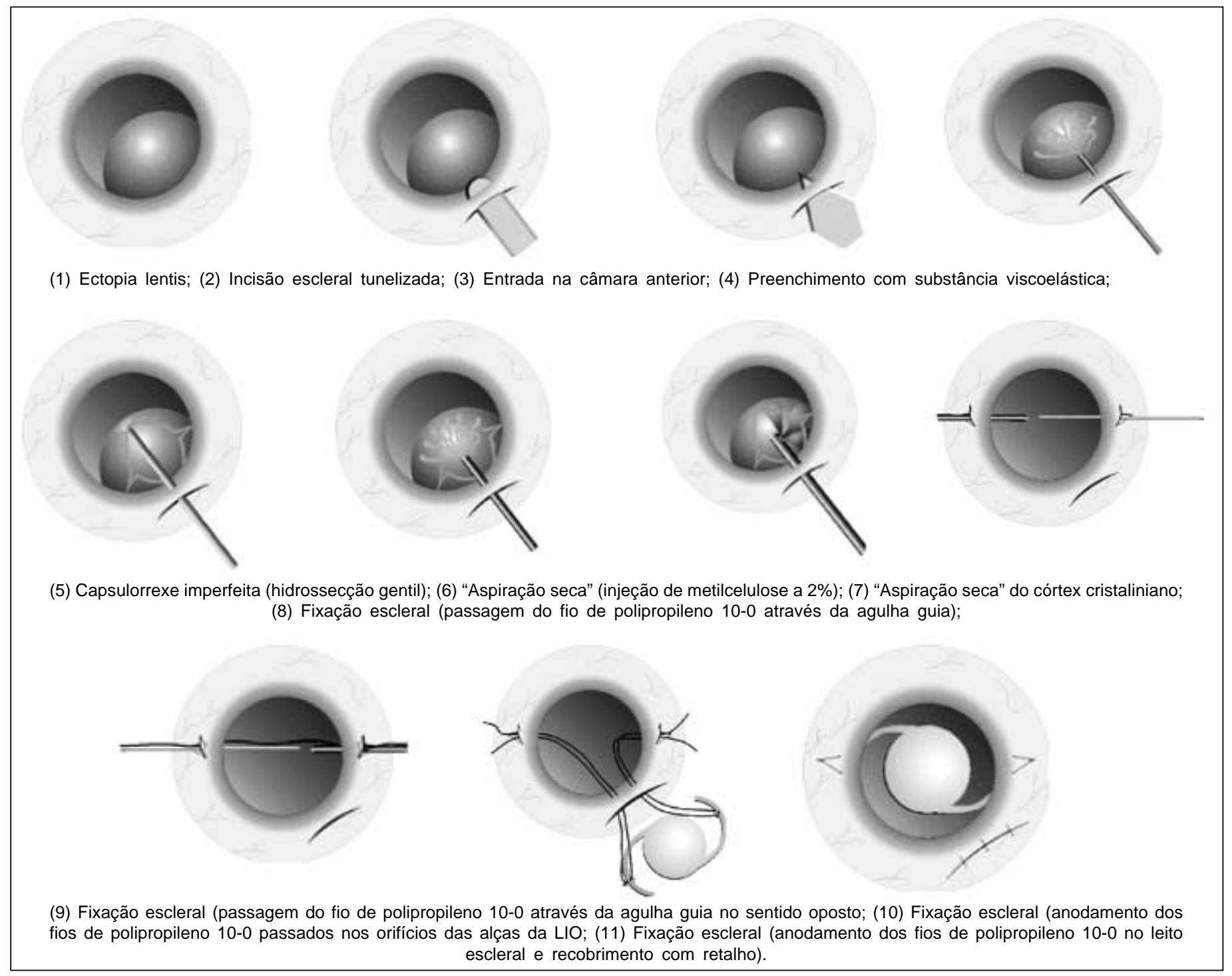

Figura 4 - Implantação da lente intra-ocular (LIO) com fixação escleral

\begin{tabular}{|lcccc|}
\hline \multicolumn{4}{|c|}{ Tabela 1. Acuidade visual sem correção no período pré-operatório } \\
e na evolução pós-operatória
\end{tabular}

ratório), no que se refere à acuidade visual sem correção, o aumento deste valor foi de 8,12 vezes para os olhos com implante de LIO por fixação e de 5,85 vezes para aqueles que receberam ANEL e LIO. No que se refere à acuidade visual com correção, estes valores aumentaram 4,46 vezes para os olhos com implante de LIO por fixação e de 3,12 vezes para aqueles que receberam ANEL e LIO. Estes números revelam, portanto, um aumento maior da acuidade visual sem $(39,0 \%) \mathrm{e}$ com $(43,0 \%)$ correção do pré-operatório para o $180^{\circ}$ dia pósoperatório nos olhos cuja LIO foi implantada por fixação.

Para se compararem três componentes passíveis de análise, ou seja, diagnóstico, subluxação do cristalino e colocação com ANEL ou fixação de LIO, e estabelecer qual deles contribuiu com a maior variação na acuidade visual, foi realizada análise de variância da acuidade visual em três pares de fatores (Tabela 4), a qual evidenciou que o grau de subluxação do cristalino ocupa o primeiro lugar na variação da acuidade visual sem correção, seguida da colocação da LIO com ANEL ou da sua fixação. O tipo de afecção (ectopia lentis simples ou síndrome de Marfan) constitui o fator que menos influenciou a variação das médias de acuidade visual sem correção. Já na 


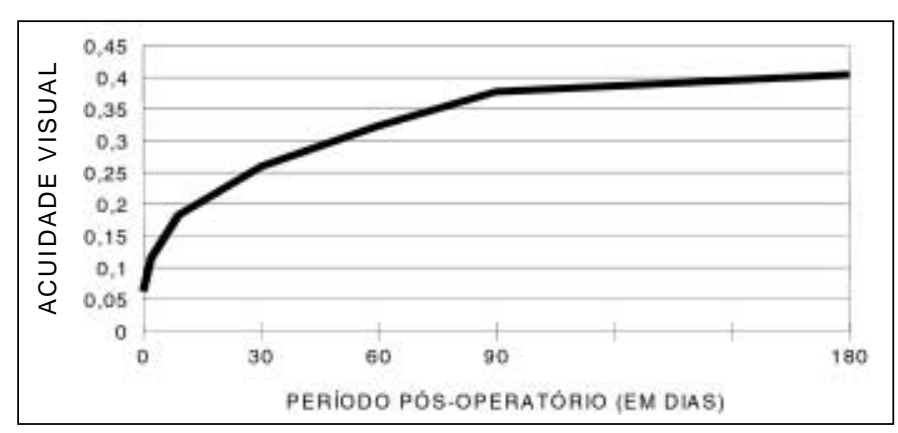

Gráfico 1 - Médias aritméticas dos valores de acuidade visual sem correção no período pré-operatório e pós-operatório (dias)

variação da acuidade visual com correção, embora a subluxação do cristalino mantenha o primeiro lugar, é imediatamente seguida pelo tipo de afecção e, só depois, pelo procedimento de colocação de ANEL ou fixação da LIO.

\section{DISCUSSÃO}

A opção pelo tratamento cirúrgico dos 51 olhos (28 pacientes) apresentados neste estudo baseou-se no fato de que a totalidade dos pacientes apresentava limite visual máximo de 0,4 ou menos, com o uso da melhor correção atualizada. Outra razão secundária referiu-se ao fato de que, para atingir a acuidade visual referida, estes pacientes utilizavam, na maior parte das vezes, elevados graus de correção que, ainda assim, não ofereciam resultado visual adequado às necessidades básicas dos pacientes.

Além da acuidade visual insatisfatória, a maioria dos pacientes apresentava alterações marcantes não só no humor, mas também nos relacionamentos pessoais e interesses, fatores esses que estavam afetando substancialmente a sua qualidade de vida, ratificando a indicação cirúrgica ${ }^{(25)}$.
Optamos por um procedimento seguro, que não repetisse as complicações do passado, que fosse reprodutível, que oferecesse fácil aprendizado aos colegas oftalmologistas interessados nesta área, e que fosse adequado à complexidade da condição estudada, sem a necessidade de empregar aparelhos cuja tecnologia implicasse em custos elevados ${ }^{(2)}$.

A técnica escolhida para o tratamento cirúrgico da ectopia lentis foi a de túnel escleral com válvula corneal, capsulorrexe, remoção manual do conteúdo cristaliniano pelo método de aspiração "seca", implantação do ANEL com fixação escleral e implantação da LIO de câmara posterior no saco capsular. Nos casos em que a capsulorrexe não foi adequada, realizamos remoção manual do conteúdo cristaliniano com aspiração "seca", implantação e fixação escleral da LIO de câmara posterior.

Utilizamos incisão conjuntival com base no fórnix, incisão escleral tunelizada auto-selante localizada, quando possível, na direção do deslocamento do cristalino, e duas incisões límbicas de serviço. O fato de essas incisões serem tunelizadas e auto-selantes permite o manuseio intra-ocular sem vazamentos e, conseqüentemente, sem flutuações no segmento anterior. Também possibilita que as manobras cirúrgicas sejam realizadas com maior segurança ${ }^{(3)}$.

Além de facilitar a exposição, a localização do túnel escleral na direção do deslocamento cristaliniano tem a vantagem de afastar-se prudentemente dos locais em que a fragilidade ou ausência zonular ou a hialóide anterior estão expostas. A ausência de integridade zonular freqüentemente dificulta $\mathrm{o}$ início da capsulorrexe em decorrência da instabilidade do cristalino. É por isso que utilizamos um cistítimo feito com agulha de $13,5 \times 4$, com extremidade angulada, com o qual realizamos a perfuração inicial na cápsula anterior do cristalino, pressionando posteriormente e complementando a capsulorrexe com pinça de Utrata. Salientamos a importância de se obter capsulorrexe que esteja o mais centralizada possível em relação ao cristalino, que seja contínua e que não ultrapasse $4 \mathrm{~mm}$ de

\begin{tabular}{|c|c|c|c|c|c|c|c|}
\hline \multirow{2}{*}{$\begin{array}{l}\text { Período de } \\
\text { avaliação }\end{array}$} & \multirow{2}{*}{$\begin{array}{l}\text { Fixação } \\
\text { escleral }\end{array}$} & \multirow{2}{*}{$\begin{array}{l}\text { ANEL e } \\
\text { LIO }\end{array}$} & \multirow{2}{*}{$\begin{array}{c}\text { Grupo } \\
\text { Geral }\end{array}$} & \multicolumn{2}{|c|}{$t$ de Student } & \multicolumn{2}{|c|}{ Mann-Whitney } \\
\hline & & & & $t$ & $\mathbf{p}$ & U & $p$ \\
\hline Pré-operatório & 0,0401 & 0,0786 & 0,0628 & 2,34 & $0,023632^{*}$ & 213,5 & 0,052073 \\
\hline $2^{\circ}$ dia de PO & 0,0817 & 0,1391 & 0,1155 & 2,08 & $0,042672^{*}$ & 194,0 & $0,020575^{*}$ \\
\hline 9ํ dia de PO & 0,1335 & 0,2178 & 0,1831 & 2,13 & $0,038016^{*}$ & 195,0 & $0,021644^{*}$ \\
\hline $30^{\circ}$ dia de $\mathrm{PO}$ & 0,1915 & 0,3064 & 0,2591 & 2,50 & $0,015886^{*}$ & 186,0 & $0,013557^{*}$ \\
\hline $60^{\circ}$ dia de $\mathrm{PO}$ & 0,2504 & 0,3747 & 0,3235 & 2,56 & $0,013734^{*}$ & 186,5 & $0,013924^{*}$ \\
\hline $90^{\circ}$ dia de PO & 0,2980 & 0,4333 & 0,3776 & 2,48 & $0,016444^{*}$ & 198,5 & $0,025775^{*}$ \\
\hline $180^{\circ}$ dia de PO & 0,3255 & 0,4600 & 0,4046 & 2,39 & $0,020775^{*}$ & 199,0 & $0,026418^{*}$ \\
\hline
\end{tabular}

\begin{tabular}{|c|c|c|c|c|c|c|c|}
\hline \multirow{2}{*}{$\begin{array}{l}\text { Período de } \\
\text { avaliação }\end{array}$} & \multirow{2}{*}{$\begin{array}{l}\text { Fixação } \\
\text { escleral }\end{array}$} & \multirow{2}{*}{$\begin{array}{l}\text { ANEL e } \\
\text { LIO }\end{array}$} & \multirow{2}{*}{$\begin{array}{c}\text { Grupo } \\
\text { Geral }\end{array}$} & \multicolumn{2}{|c|}{ t de Student } & \multicolumn{2}{|c|}{ Mann-Whitney } \\
\hline & & & & $t$ & p & U & $\mathbf{p}$ \\
\hline Pré-operatório & 0,1202 & 0,2209 & 0,1795 & 3,29 & $0,001865^{*}$ & 165,5 & $0,004222^{*}$ \\
\hline $180^{\circ}$ dia de $\mathrm{PO}$ & 0,5363 & 0,6883 & 0,6257 & 2,07 & $0,043455^{\star}$ & 204,0 & $0,033642^{*}$ \\
\hline
\end{tabular}




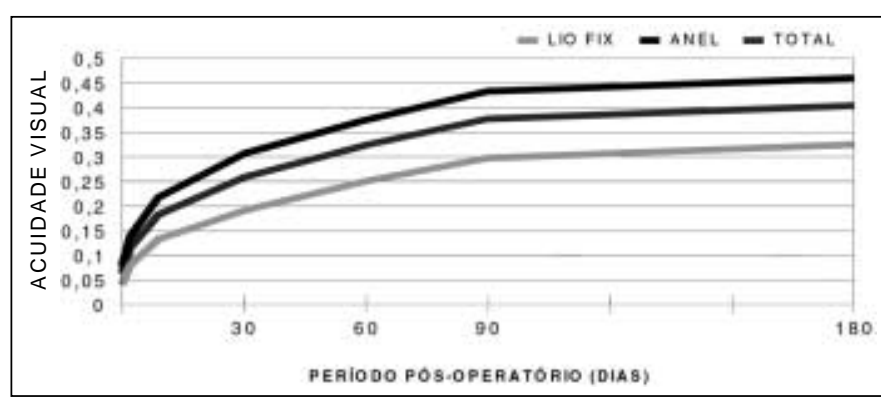

Gráfico 2 - Médias relativas à acuidade visual sem correção, de acordo com o procedimento utilizado para implante da LIO, no período préoperatório e na evolução pós-operatória

diâmetro ${ }^{(26)}$. Estes quesitos são necessários para que se possa implantar o anel endocapsular com segurança.

Os casos estudados apresentavam defeitos zonulares preexistentes, motivo pelo qual optamos por implantar o anel endocapsular previamente à aspiração do conteúdo sacular, de modo a assegurar maior estabilidade, a restabelecer parcialmente a centralização (evitando o prolapso anterior do vítreo), e a evitar estender a diálise zonular do cristalino subluxado.

A remoção do conteúdo cristaliniano foi realizada pelo método de irrigação e aspiração "seca". Assim, a metilcelulose era injetada no saco capsular, e o conteúdo cristaliniano, de consistência mole, era aspirado por uma cânula de $0,4 \mathrm{~mm}$, em manobras repetidas e sucessivas. Acreditamos que este método apresente vantagens sobre a facoemulsificação ou facoaspiração ou, ainda, aspiração com cânula de dupla via, pois, sem a infusão de solução salina balanceada, o vítreo não é conseqüentemente hidratado, assim como há menor flutuação intra-ocular. A presença constante de metilcelulose mantém a pressurização intra-ocular, tamponando a área de fragilidade zonular, o que acarreta maior proteção quanto à herniação anterior do vítreo.

A implantação da LIO e a fixação escleral de um de seus hápticos em conjunto com o anel endocapsular asseguram centralização mais segura ${ }^{(4-5,12,27-30)}$.

Há casos em que a subluxação cristaliniana pode estar muito acentuada, o que, aliado à ausência ou fraqueza zonular de grande extensão, pode somar dificuldades que impedem a realização de capsulorrexe em padrão ideal, devendo-se evitar o implante do anel endocapsular. Nestas situações, utiliza-se a mesma técnica de irrigação e aspiração "seca" do conteúdo cristaliniano com uso de metilcelulose. Todavia, a ausência de anel endocapsular dificulta a realização de aspiração "seca" do cristalino, pois o saco capsular é freqüentemente distorcido durante a aspiração, obrigando a interrupção momentânea da manobra para reposição de metilcelulose, de modo que reassuma a sua forma e mantenha o tamponamento da área de fraqueza zonular ${ }^{(4-5)}$.

Com relação aos resultados alcançados na correção cirúrgica da ectopia lentis tal qual aqui proposta, observamos decréscimo do valor de desvio padrão em relação ao da média da acuidade visual ao longo da evolução pós-operatória, o que indica que a acuidade visual na fase cirúrgica final é mais homogênea do que no pré-operatório.

A média da acuidade visual com correção no $180^{\circ}$ dia pósoperatório foi de aproximadamente 3,5 vezes o valor médio inicial ao pré-operatório, que correspondeu a cerca de $28,7 \%$ do valor médio registrado seis meses após a operação.

As médias relativas à acuidade visual sem e com correção foram significantemente mais altas para os olhos em que se colocou ANEL e LIO, comparados com os olhos que tiveram a fixação escleral da LIO, tanto no período pré-operatório quanto no $180^{\circ}$ dia pós-operatório. Todavia, quando se comparou a variação entre os valores iniciais pré-operatórios e os valores finais pós-operatórios, os olhos com fixação escleral da LIO apresentaram acuidade visual proporcionalmente maior do que os olhos com ANEL e LIO. Esta aparente discrepância se justifica pelo fato de os valores da acuidade visual terem sido, já no pré-operatório, casualmente menores no grupo de olhos que veio a receber a lente intra-ocular com fixação escleral. Acreditamos que este achado possa se modificar em estudos com maior número de indivíduos e com maior tempo de seguimento.

A variação dos valores médios relativos à acuidade visual sem correção do grupo como um todo, registrados nas diferentes avaliações pré e pós-operatórias, evidenciou diferenças significativas na grande maioria das combinações de pares avaliadas, exceção feita para as variações registradas entre o pré-operatório e segundo dia pós-operatório; entre o $60^{\circ}$ e o $90^{\circ}$ dia pós-operatório; e entre o $90^{\circ}$ e o $180^{\circ}$ dia pós-operatório. A menor variação foi observada entre o $90^{\circ}$ e o $180^{\circ}$ dia pós-operatório. Certamente, a pequena variação registrada entre o pré-operatório e o segundo dia pós-operatório deveuse ao fato de o procedimento operatório, per se, induzir acentuado astigmatismo nos primeiros dias após a cirurgia, além de, clinicamente, ocasionar algum grau de edema corneal e edema cistóide macular ${ }^{(14)}$, que tendem a melhorar com o passar dos dias.

A variação dos valores médios da acuidade visual sem e com correção registrados nas diferentes avaliações pré e pós-

\begin{tabular}{|c|c|c|c|c|}
\hline Acuidade visual & Período de observação & $\begin{array}{c}\text { Graus de subluxação } x \\
\text { ANEL/fixação }\end{array}$ & $\begin{array}{c}\text { Graus de subluxação } x \\
\text { diagnóstico }\end{array}$ & $\begin{array}{l}\text { Anel/fixação } x \\
\text { diagnóstico }\end{array}$ \\
\hline Sem correção & $\begin{array}{c}\text { Pré-operatório } \\
2^{\circ} \text { ao } 180^{\circ} \text { dia PO }\end{array}$ & $0,004164^{*}$ & $0,000021^{*}$ & $0,018479^{*}$ \\
\hline Com correção & $\begin{array}{c}\text { Pré-operatório } \\
2^{\circ} \text { ao } 180^{\circ} \text { dia PO }\end{array}$ & $\begin{array}{c}\left(0,026548^{*}\right) \\
0,017364^{*}\end{array}$ & $\begin{array}{l}0,037288^{*} \\
0,000585^{*}\end{array}$ & $\begin{array}{c}\left(0,004741^{*}\right) \\
0,046119^{*}\end{array}$ \\
\hline
\end{tabular}


operatórias foi enfaticamente influenciada pelo grau de subluxação do cristalino: quanto maior é o grau de subluxação cristaliniana, maior a complexidade na correção óptica, e maior a possibilidade de distúrbios no desenvolvimento sensorial, ou seja, ambliopia. Por isso, este trabalho evidencia que a acuidade visual média, com e sem correção, tanto no período pré como no pós-operatório, é maior no grau 1 de subluxação, decrescendo progressivamente nos graus 2 e 3 .

O tipo de procedimento cirúrgico (ANEL e LIO ou fixação escleral da LIO) influenciou mais do que o diagnóstico de base sobre a variação dos valores médios de acuidade visual sem correção. Contrariamente, a variação dos valores médios de acuidade visual com correção foi mais influenciada pelo diagnóstico do que pelo tipo de procedimento operatório.

Os olhos em que foi possível o implante do anel endocapsular foram aqueles cuja subluxação cristaliniana não era tão extrema, tanto que a capsulorrexe foi realizada idealmente. Além disso, menores níveis de ambliopia foram identificados entre estes pacientes com cristalinos menos subluxados.

Acreditamos que o implante do ANEL e LIO criam melhores condições de posição e estabilidade da LIO, permitindo melhor acuidade visual sem correção.

Por outro lado, o diagnóstico influencia mais do que o tipo de procedimento nos valores médios de acuidade visual com correção porque, com relação à visão, os olhos dependem diretamente da qualidade de desenvolvimento sensorial. Assim, dados os sinais e sintomas da síndrome de Marfan, os olhos destes pacientes têm menor potencial de desenvolvimento sensorial do que os olhos com ectopia lentis simples. Portanto, a importância do procedimento operatório utilizado é menor para o desenvolvimento da visão, uma vez que o erro refracional resultante será corrigido.

Ressalte-se que não foi possível apenas a diminuição da dependência de correções ópticas de elevadas dioptrias, mas também o foi o restabelecimento da acuidade visual daqueles olhos que, no pré-operatório, apresentavam subluxações em que o equador cristaliniano já se encontrava no meio da pupila, e, igualmente, dos olhos que apresentavam torções do cristalino sobre o seu eixo gerando graus elevados de astigmatismo. A recuperação visual com menor vício de refração trouxe, inclusive, maior impacto na qualidade de vida para os pacientes operados, conforme atestado na avaliação subjetiva da satisfação com a cirurgia realizada pelos próprios pacientes ${ }^{(25)}$.

Importante citar que a acuidade visual pós-operatória foi inversamente proporcional ao comprimento axial nos 51 olhos estudados. Quanto maior o comprimento axial, maior seria o erro refracional e, portanto, maior a chance de desenvolvimento de ambliopia. Alguns autores já haviam observado que medidas maiores de comprimento axial estavam intimamente relacionadas com ambliopia e diminuição da acuidade visual ${ }^{(25,31)}$.

A implantação da correção óptica (LIO) na cirurgia da ectopia lentis é essencial para a obtenção de acuidade visual satisfatória. Nos pacientes com subluxação cristaliniana, a dificuldade refere-se ao suporte capsular satisfatório para que se atinja posicionamento adequado da área óptica da LIO. Nesta série, este objetivo foi atingido com implantação de anel endocapsular e LIO, quando possível, ou com fixação escleral da LIO. Contudo, apesar dos esforços, quatro olhos apresentaram pequenos deslocamentos da área óptica que, todavia, não comprometeram a acuidade visual nem a satisfação do paciente, exceção feita a um caso que teve a LIO fixada na esclera, conforme o protocolo estabelecido, mas evoluiu com subluxação da lente implantada, que obrigou a sua refixação cirúrgica. Não houve qualquer intercorrência intra-operatória neste caso, e o paciente não referiu qualquer trauma ou esforço excessivo, não ficando, deste modo, evidente o motivo desta ocorrência.

Mais do que apenas a melhora da acuidade visual mensurada de modo objetivo, trata-se de procedimento terapêutico que releva, antes, a função visual como um todo funcionando como instrumento facilitador para a execução de atividades de vida diária de modo mais prazeroso e eficaz, aumentando, assim, o interesse, o entusiasmo e a auto-estima do paciente com relação ao seu próprio cotidiano ${ }^{(24)}$. Os dados aqui reunidos permitem, portanto, afirmar que o tratamento cirúrgico da ectopia lentis pode ser considerado nas situações de insatisfação com a acuidade visual obtida com correção. Se realizada antes de subluxação importante, ou seja, em olhos que apresentem graus 1 e 2, os resultados serão mais satisfatórios, assim como a técnica cirúrgica será mais completa e segura, resultando em índice elevado de recuperação visual e satisfação dos pacientes ${ }^{(25)}$.

\section{CONCLUSÕES}

Mediante as técnicas operatórias apresentadas, a correção cirúrgica da ectopia lentis simples ou associada com a síndrome de Marfan é segura e eficaz, com recuperação significante da acuidade visual, embora os resultados operatórios dependam, intrinsecamente, dos graus pré-operatórios de subluxação do cristalino.

\section{ABSTRACT}

Purpose: To assess the results as to visual acuity of two different surgical procedures for ectopia lentis. Methods: Fifty-one eyes of 28 patients ( 16 males and 12 females, mean age $16.00 \pm$ 8.5) with simple (19 cases) or Marfan syndrome-associated (9 cases) ectopia lentis with different levels of subluxation underwent lens extraction with implantation of intraocular lenses (IOL) with scleral fixation (21 cases) or by lens extraction with implantation of the intraocular lenses in the capsular bag expanded by endocapsular ring (RING) (30 cases). Result analysis emphasized pre- and postoperative visual acuity during a six-month follow-up. Results: Both techniques showed sig- 
nificant increase of postoperative visual acuity with and without correction, but it was better among the cases operated on by scleral fixation of the intraocular lenses. More than the used technique, the preoperative subluxation grades were crucial as to the results. Conclusion: The two surgical techniques for correction of simple or Marfan syndrome-associated ectopia lentis are safe and effective, resulting in significant recovery of visual acuity, although surgical results are completely dependent on the preoperative lens subluxation grades.

Keywords: Ectopia lentis/surgery; Marfan syndrome; Lenses intraocular; Sclera/surgery; Lens capsule, crystalline/surgery; Prostheses and Implants; Visual acuity; Adolescent

\section{REFERÊNCIAS}

1. Adank AM, Hennekes R. Phacoemulsification of the subluxated or atopic lens. Bull Soc Belge Ophthalmol. 1993;249:33-9.

2. Blumenthal M, Kurtz S, Assia EI. Hidroexpression of subluxated lenses using a glide. Ophthalmic Surg. 1994;25(1):34-7.

3. Waiswol M, Cohen R, Ejzenbaum F. Técnica de "mini-nuc" de Blumenthal: resultados de 454 casos. Arq Bras Oftalmol. 2001;64(3):217-21.

4. Gimbel HV, Basti S, Ferensowicz M, DeBroff BM. Results of bilateral cataract extraction with posterior chamber intraocular lens implantation in children. Ophthalmology. 1997;104(11):1737-43.

5. Cionni RJ, Osher RH. Management of profound zonular dialysis or weakness with a new endocapsular ring designed for scleral fixation. J Cataract Refract Surg. 1998; 24(10):1299-306.

6. Sun R, Gimbel HV. In vitro evaluation of the efficacy of the capsular tension ring for managing zonular dialysis in cataract surgery. Ophthalmic Surg Lasers. 1998;29(6):502-5.

7. Faria MAR, Oliveira TL. Implante secundário de lente intra-ocular: estudo de 23 casos. Rev Bras Oftalmol. 1999;58(4):263-8.

8. Gimbel HV, Sun R. Role of capsular tension rings in preventing capsule contraction [letter]. J Cataract Refract Surg. 2000;26(6):791-2.

9. Maumenee IH. The eye in the Marfan syndrome. Trans Am Ophthalmol Soc. 1981;79:684-733

10. Nelson LB, Maumenee IH. Ectopia lentis. Surv Ophthalmol. 1982;27(3):143-6.

11. Ruellan YM. [Surgical treatment of dislocation of the crystalline lens]. J Fr Ophthalmol. 1985;8(12):823-31. French.
12. Osher RH. Synthetic zonula offers hope in tough cataract cases. Ophthalmol Times Int. 1998; Jan/Feb:8-11.

13. Zetterström C, Lundavall A, Weeber H Jr, Jeeves M. Sulcus fixation without capsular support in children. J Cataract Refract Surg. 1999;25(6):776-81.

14. Salehpour O, Lavy T, Leonard J, Taylor D. The surgical management of nontraumatic ectopic lenses. J Pediatr Ophthalmol Strabismus. 1996;33(1):8-13.

15. Seetner AA, Crawford JS. Surgical correction of lens dislocation in children. Am J Ophthalmol. 1981;91(1):106-10.

16. Syrdalen P. Pars plana technique for removal of congenital subluxated lenses in young patients. Acta Ophthalmol (Copenh). 1987;65(5):585-8.

17. Behki R, Noel LP, Clarke WN. Limbal lensectomy in the management of ectopia lentis in children. Arch Ophthalmol. 1990;108(6):809-11.

18. Plager DA, Parks MM, Helveston EM, Ellis FD. Surgical treatment of subluxated lenses in children. Ophthalmology. 1992;99(7):1018-21; discussion p.1022-3.

19. Marcus DM, Topping TM, Frederick Jr AR. Vitreoretinal management of traumatic dislocation of the crystalline lens. Int Ophthalmol Clin. 1995;35(1): 139-50.

20. Yu YS, Kang YH, Lim KH. Improvements in visual acuity following limbal lensectomy for subluxated lenses in children. Ophthalmic Surg Lasers. 1997;28 (12):1006-10.

21. Harrison DA, Mullaney PB, Mesfer SA, Awad AH, Dhindska H. Management of ophthalmic complications of homocystinuria. Ophthalmology. 1998;105(10): 1886-90.

22. Siganos DS, Siganos CS, Popescu CN, Margaritis VN. Clear lens extraction and intraocular lens implantation in Marfan's syndrome. J Cataract Refract Surg. 2000;26(5):781-4.

23. Severo NS, Kleinert F, Kwitko S. Conduta cirúrgica na subluxação do cristalino. Arq Bras Oftalmol. 2004;67(1):9-12.

24. Centurion V, Lacava AC, Caballero JC, Modé E. Anel intracapsular na cirurgia da catarata. Rev Bras Oftalmol. 1999;58(4):641-4.

25. Waiswol M. Contribuição ao tratamento cirúrgico da ectopia lentis [tese]. São Paulo: Faculdade de Medicina da Universidade de São Paulo; 2003.

26. Rosenthal KJ. When and how to use the Morcher capsular tension ring. Rev Ophthalmol 1998;11(3):105-12.

27. Hara T, Hara T, Yamada Y. "Equator ring" for maintenance of the completely circular contour of the capsular bag equator after cataract removal. Ophthalmic Surg. 1991;22(6):358-9.

28. Nagamoto T, Bissen-Miyajima H. A ring to support the capsular bag after continuous curvilinear capsulorhexis. J Cataract Refract Surg 1994;20(4):417-20.

29. Legler U. The capsular ring: a new device for complicated cataract surgery [filme]. In: $3^{\circ}$ International Congress of Cataract, IOL and Refractive Surgery, 812 May 1993, Seattle, Washington.

30. Hara T, Hara T, Yamada Y. Equatorial ring for maintenance of the completely circular contour of the capsular equator, to prevent opacification of the posterior capsule after cataract removal [filme]. In: $3^{\circ}$ International Congress of Cataract, IOL and Refractive Surgery, 8-12 May 1993, Seattle, Washington.

31. Rasooly R, BenEzra D. Congenital and traumatic cataract. The effect on ocular axial length. Arch Ophthalmol. 1988;106(8):1066-8.

\section{Congresso dos Centros de Ensino em Oftalmologia do Paraná}

\section{3 a 05 Novembro Curitiba - PR}

INFO RMA ÇÕ ES: Tels. (41) 3024-6065/9996-6284

E-mail: eventos@authentictour.com.br 